\title{
OTIOTOMICS
}

Revista de economía, empresa y sociedad

Dosier «Vectores de sostenibilidad: visiones desde la economía»

Coordinador: Albert Puig Gómez

UN DEBATE ABIERTO

\section{Contradicciones y promesas de la economía circular}

\section{Zora Kovacic}

Universitat Oberta de Catalunya

RESUMEN La economía circular es una alternativa al modelo linear de «extracción-uso-desecho» de los recursos naturales, que se sustituye por un modelo circular de «reciclar, reutilizar y reparar». La economía circular promete convertir las preocupaciones por la sostenibilidad en sinergias que promueven el crecimiento económico: en vez de proponer frenar la actividad económica para utilizar menos recursos naturales y generar menos residuos y emisiones, los nuevos modelos de negocios circulares supuestamente permitirían seguir utilizando los recursos que se necesitan minimizando el impacto ambiental. A pesar del gran entusiasmo generado por esas promesas en las políticas públicas, la idea de una economía circular es también fuente de mucha controversia a nivel científico, ya que la idea de que es posible recircular la mayoría de los materiales y productos va en contra de las leyes de la termodinámica. Entonces, ¿por qué una idea que genera tanta controversia ha tenido, y sigue teniendo, tanto éxito en las políticas públicas? Durante la crisis económica de la década de 2010, la economía circular podía verse como una oportunidad de seguir legislando en favor del medio ambiente en un momento en el que la crisis económica, el desempleo y la deuda eran las preocupaciones principales. Diez años después, la economía circular se ha convertido en una de las políticas estrella del «Pacto Verde Europeo» y está cada vez más presente en todos los ámbitos, incluyendo las políticas urbanas. En el contexto actual, las sinergias que promete la economía circular pueden ofuscar los retos de la sostenibilidad y crear un debate apolítico.

PALABRAS CLAVE entropía; desaceleración; sostenibilidad; políticas públicas; sinergias; controversia 


\section{Contradictions and promises of circular economy}

ABSTRACT Circular economy is an alternative to the linear "extract-use-dispose" economic model, based on the ideas of recycling, repair and reuse. The circular economy promises to turn the trade-offs of unsustainable economic growth in a limited planet into synergies that make sustainability a growth strategy. The knowledge base of the circular economy, however, is highly divided and characterised by controversy over the very possibility of circularity itself. This paper asks why such a controversial idea has gained so much traction in public policies, at the EU level and at lower scales, such as the urban level. Although the focus on win-win solutions was understandable in the context in which the first circular economy policies were formulated in the EU, in the current context this policy framing may obfuscate more than it enlightens by rendering debates about complex sustainability issues technical and apolitical.

KEYWORDS entropy; deacceleration; sustainability; public policies; synergies; controversy

\section{Introducción}

La economía circular nace como alternativa a la economía lineal, con el objetivo de cambiar el modelo «extracción-uso-desecho" de los recursos naturales por un modelo más sostenible que pone el acento en reciclar, reutilizar y reparar, para minimizar la extracción de recursos naturales escasos y no renovables y minimizar la generación de residuos. La economía circular parecería, a primera vista, reconciliar la tensión entre crecimiento económico y sostenibilidad: en vez de proponer frenar la actividad económica para utilizar menos recursos y generar menos residuos y emisiones, nuevos modelos de negocios circulares podrían permitir seguir utilizando los recursos que se necesitan sin impacto ambiental, ya que los recursos existentes pueden ser «recirculados», prescindiendo parcialmente del medio ambiente, y por lo tanto protegiéndolo. A pesar del gran entusiasmo generado por esas promesas en las políticas públicas, la idea de una economía circular es también fuente de mucha controversia a nivel científico. La economía circular es un caso de estudio muy especial en este sentido, ya que la idea de base (la posibilidad de recircular la mayoría de los materiales y productos) no tiene respaldo en las teorías científicas y se sostiene sobre todo en promesas de innovaciones e invenciones, en visiones para el futuro y en el deseo de ser más sostenibles.

He tenido la oportunidad de trabajar en este tema entre 2016 y 2020, dentro del marco del proyecto H2020 MAGIC (Moving towards Adaptive Governance In Complexity), en el que se estableció una estrecha colaboración con algunas instituciones de la Comisión Europea y se realizaron entrevistas a los artífices de las políticas para la economía circular. Cuando empezamos a analizar el concepto y las políticas de economía circular en 2016, nuestro objeto de investigación era una política en formación, una serie de principios más que una política ya formada, dado que la primera comunicación oficial sobre la economía circular se publicó en 2014. Ahora, la economía circular es un elemento central en el «Pacto Verde Europeo», la nueva estrategia de crecimiento de la Unión Europea (UE) y está cada vez más presente también en las políticas de los países miembros de la UE, así como en otras escalas de gobernanza, como en las políticas urbanas. En mi opinión, las contradicciones a la base teórica de la economía circular siguen sin resolverse y representan en cierto modo una de las contradicciones del capitalismo, que en la medida que crece destruye los recursos necesarios para su propia reproducción.

\section{1. ¿La economía puede ser circular?}

Una de las características más particulares e interesantes de la economía circular es, en mi opinión, la controversia científica que hay por detrás de la idea de circularidad, que según algunos es una imposibilidad y según otros, la clave de un futuro más sostenible. Hay varias críticas a la idea de circularidad, aquí resalto dos. 


\subsection{Los ritmos de la economía}

Una de las claves del éxito del capitalismo ha sido la linealización de los procesos y la aceleración del crecimiento económico que eso ha permitido (Giampietro, 2019). La linealización se puede observar en la preferencia por recursos no renovables antes que renovables, como puede ser el petróleo antes que las energías renovables. El petróleo no requiere ser producido, sino simplemente extraído. La extracción de petróleo no está limitada por la radiación solar o el viento disponible, por los tiempos de cultivo de la biomasa, por la estacionalidad, etc. Eso permite una aceleración de los procesos económicos según las lógicas del capital: si la demanda crece, aumenta la extracción. La circularidad sería algo como internalizar tanto los procesos como los tiempos de producción de los recursos en la economía. Internalizar los procesos de producción de recursos primarios significaría crear una economía mucho más lenta (Kovacic et al., 2019). Como escriben Murray et al., "se requieren 9 días para que el agua circule en la atmosfera, y 37.000 años para que los océanos completen un ciclo. El fósforo requiere 2000 años para recircular en el suelo. El dióxido de carbono lleva 4 años para circular en la atmósfera y el oxígeno 3,7 millones de años» (Murray et al., 2017; traducción de la autora). No es lo mismo usar fertilizantes producidos gracias a los combustibles fósiles que esperar la regeneración de fósforo y nitrógeno según los ciclos naturales.

La linealización hace que el ritmo de la actividad económica sea determinado por la capacidad de producción de bienes y servicios, mientras que una economía circular dependería del ritmo de regeneración de los recursos primarios que se necesitan para la producción de bienes. Además, internalizar los procesos de generación y regeneración de la naturaleza tendría un coste muy elevado. La historia de éxito de la economía está correlacionada con el aprovechamiento de los gradientes energéticos (Hall y Klitgaard, 2011), que es aprovechar el potencial que se genera por otros agentes con un mínimo coste para usarlos como motor económico. Los biocombustibles o el reciclaje son precisamente una forma de internalizar esos procesos y acabar con la gran ventaja de dejar que la naturaleza se encargue de todo eso tan costoso. Por lo tanto, aquí está la primera contradicción de la idea de la economía circular como estrategia de crecimiento económico: en la medida en que la economía se puede «recircular», esta estrategia llevaría a procesos de producción mucho más lentos, más costosos y a la adaptación del crecimiento económico a la capacidad regenerativa de la economía. La economía circular podría ser más bien una estrategia de decrecimiento y desaceleración.

La desaceleración es una lectura muy poco ortodoxa de lo que significa «economía circular» pero posiblemente es también un camino hacia la sostenibilidad. Justamente la preferencia por recursos no renovables (Giampietro et al., 2011) es uno de los mecanismos que crea conflictos entre el modelo económico capitalista y el medio ambiente porque se extraen recursos más rápidamente de lo que se regeneran y, por lo tanto, hay una desalineación de los ciclos económicos del capitalismo y de los ciclos ecosistémicos. De hecho, el hecho de calificar una actividad como renovable o no renovable se puede remontar a una cuestión de ritmo: la agricultura es sostenible cuando también se respetan los ciclos de regeneración del suelo, del fósforo, del nitrógeno, de recarga de los acuíferos, etc. El aumento de la producción agrícola a menudo supone aumentar el ritmo de producción y puede llevar a la degradación física de los suelos, el agotamiento de sus nutrientes, la sobreexplotación de los acuíferos, etc. Una economía sostenible se podría conceptualizar como una economía ajustada a los ritmos de la naturaleza, donde el objetivo deja de ser el crecimiento económico y pasa a ser el equilibrio entre el sistema económico y el sistema ecológico que lo sostiene.

Por otro lado, hay una correlación entre el crecimiento poblacional y la aceleración en el uso de los recursos naturales (Giampietro et al., 2011; Hall y Klitgaard, 2011). Esa correlación crea una situación de lock-in: para sostener a la población mundial es necesario usar una cantidad insostenible de recursos naturales y no está claro si una agricultura sostenible, ecológica y supeditada al ritmo de regeneración de los recursos renovables podría producir alimentos suficientes para alimentar a una población creciente (de humanos y ganado). La sostenibilidad va más allá de la discusión de la cantidad de recursos que se usan y del ritmo de extracción de esos recursos, y requiere también tener en consideración la identidad y las características de lo que se quiere sostener: en este caso, una población humana en crecimiento y sus patrones de consumo. 


\subsection{La economía como proceso entrópico}

Otro importante objetivo de la economía circular es el de minimizar los desechos de materiales y emisiones. En la medida en que crece la conciencia de los impactos ambientales y climáticos de la actividad económica, las políticas públicas de más y más países, incluyendo los de la Comisión Europea, están incorporando medidas para la reducción de los residuos y de las emisiones de gases de efecto invernadero. Recircular los materiales y los productos es potencialmente una manera de reducir residuos. Pero ¿hasta qué punto se pueden reducir emisiones y residuos? ¿Qué tan circular puede ser la economía? Este es posiblemente el punto que más controversia ha generado, lo que ha llevado a varios autores a articular una crítica muy dura de la economía circular como algo imposible, ya que va en contra de las leyes de la termodinámica (Martínez-Alier, 2016; Mayumi y Giampietro, 2020). Los flujos de energía y de alimentos se degradan con el uso y no se pueden reciclar totalmente, solo muy parcialmente. La entropía que rige nuestro universo es un proceso irreversible que impide ser circular.

Para Herman Daly, la linealidad de la economía biofísica era algo tan evidente que llegó a criticar las representaciones circulares usadas en macroeconomía para representar el producto nacional. Típicamente, los libros de texto de economía representan el valor encapsulado en los bienes y servicios como un flujo que va de las empresas a los hogares, y un valor equivalente, encarnado en los factores de producción, que fluye de los hogares a las empresas. Se crea así un intercambio en el que el producto nacional se genera a partir de la circulación de valor en la economía. Según Daly, preguntar a un estudiante de economía que explique esta como algo circular sería tan absurdo como «preguntar a un estudiante de ingeniería que explicara cómo un coche puede moverse usando sus propios humos de escape, o preguntar a un estudiante de biología que explique cómo un organismo puede metabolizar sus propios excrementos, explicar cómo los productos reentran como insumos, o mejor dicho, insumos primarios, requiere que el estudiante descubra el secreto del movimiento perpetuo» (1985, pág. 281).

Haas et al. (2015) han estimado que los flujos de combustibles fósiles representan alrededor del $21 \%$ de la masa de los materiales que consume la economía mundial, y la biomasa, un 33 \% aproximadamente (véase tabla 1). Esto es, más de la mitad (54\%) de los flujos materiales se usa en procesos entrópicos de alta disipación. De los combustibles fósiles, un $98 \%$ se usa como energía y no puede ser reciclado. Del restante $2 \%$, hay una parte de combustibles fósiles que se destinan a la producción de plásticos, y solo parte de esa producción es actualmente reciclable. Estamos hablando de una fracción del $2 \%$ del $20 \%$ del total de los flujos de materiales, o sea, algo tan marginal que está dentro del margen de error de esas estimaciones. De la biomasa, un $80 \%$ se destina a usos energéticos, alimentarios y como alimentos para ganado. El 20 \% restante incluye materiales como el papel y la madera para la construcción, que pueden ser potencialmente reciclados. Haas y sus colegas estiman que, a nivel mundial, la tasa de circularidad de la biomasa por los humanos está alrededor del 3 \% del total de los materiales utilizados. El resto de los flujos materiales, que no son ni combustibles fósiles ni biomasa, está dominado por materiales que se destinan a la construcción, estimado por Haas y sus colegas en un $38 \%$ del total. Esos materiales se consideran como un stock, o sea, son materiales que no circulan en la economía, sino que se quedan en los edificios, en las carreteras, en las infraestructuras indefinidamente. Lo que más se recicla son los materiales metálicos, que representan apenas un $8 \%$ del total. Haas y sus colegas concluyen que la tasa de «circularidad» de la economía mundial es de un 6 \%, y en la Unión Europea de un $13 \%$.

\section{Tabla 1. Flujos de materiales usados en la economía}

\begin{tabular}{|l|c|}
\hline \multicolumn{1}{|c|}{ Tipo de flujo } & Porcentaje del consumo total de materiales a nivel mundial \\
\hline Combustibles fósiles & $21 \%$ \\
\hline Biomasa & $33 \%$ \\
\hline Materiales no metálicos & $38 \%$ \\
\hline Materiales metálicos & $8 \%$ \\
\hline
\end{tabular}

Fuente: Haas, Krausmann, Wiedenhofer y Heinz (2015) 
Estas estadísticas son una parte importante del debate sobre la economía circular y de la construcción de imaginarios colectivos sobre las promesas de la circularidad (Völker et al., 2020). La promesa de la economía circular es minimizar la entrada y salida de materiales, no eliminar completamente la dependencia del ecosistema. Las políticas públicas no hablan de llegar al 100 \% de circularidad, sino de aumentar el nivel de circularidad de la economía. En un workshop organizado con expertos de la Agencia Europea de Medioambiente en el que participé en 2018, se dijo que "nadie quisiera que la economía fuese menos circular». Es decir, la imposibilidad de llegar a un $100 \%$ de circularidad y la incompatibilidad de la idea de economía circular con los «hechos científicos» no necesariamente deslegitimizan las políticas públicas para la economía circular.

Por otro lado, cabe preguntarse: ¿hasta qué punto se pueden «minimizar los residuos»? En la Unión Europea las tasas de reciclaje de materiales metálicos son del $90 \%$, para los cristales es del $80 \%$ y para los papeles y cartón es del $65 \%$ (Eurostat, 2021). Llegar al 100 \% en esas categorías podría aumentar la tasa de circularidad total en 1 o 2 puntos porcentuales como mucho. En otras palabras, ¿es posible ser significativamente «más circulares» que un $13 \%$ ? ¿La economía circular es suficiente para alcanzar los objetivos de sostenibilidad de la Comisión Europea?

\section{Necesidades políticas}

Si hay tanta controversia sobre el concepto de circularidad, ¿por qué la idea de economía circular ha tenido, y sigue teniendo, tanto éxito en las políticas públicas? Para contestar a esta pregunta, se puede hacer el ejercicio de desenfocar la mirada de la idea en sí misma y enfocarla al contexto político en el que la idea de economía circular surgió. La primera comunicación de la Comisión Europea sobre economía circular fue publicada en 2014 como una estrategia de reducción de los residuos bajo una lógica de crecimiento sostenible. Con el cambio de comisión en 2015, la primera comunicación fue sustituida por una nueva (Comisión Europea, 2015) mucho más «ambiciosa», según los responsables, en la que la economía circular se convierte en una estrategia de crecimiento económico. Algo importante que remarcar es que 2014 y 2015 eran todavía los años en lo que la UE se estaba recuperando de la crisis económica empezada en 2008 con la burbuja de los subprime en Estados Unidos. Por lo tanto, se trataba de un contexto político muy poco favorable a las políticas ambientales o a cualquier política que cuestionara la prioridad y necesidad de reanudar el crecimiento económico. La economía circular en este contexto no significa que la economía tiene que llegar a ser (más) circular, sino que es una manera de permitir que las preocupaciones por la sostenibilidad sigan teniendo un espacio y voz en las políticas públicas. Un discurso que pidiera reducir el consumo de recursos naturales y el impacto de las actividades económicas en el medio ambiente hubiera sido rechazado.

Uno de los problemas políticos del discurso ambientalista es que suele tener un mensaje de fondo que dice «iParen!» (Kovacic et al., 2019). Paren de usar tantos recursos naturales, paren de contaminar, paren las actividades que interfieren negativamente con los ecosistemas. En un contexto en que la prioridad número uno es la de acelerar, volver a crecer y ver la economía retomar su ritmo, el mensaje «iParen!» no es bienvenido. Tampoco puede existir un discurso que intente limitar el crecimiento económico, o que hable de trade-offs, costes y beneficios, ganadores y perdedores. Lo que aporta la economía circular es un discurso donde solo hay beneficios y ganadores: recirculando los materiales escasos, o poco comunes (rare earth materials), supuestamente se supera el problema de los límites, creando modelos de negocios circulares se producen nuevos nichos de mercado que supuestamente permiten el crecimiento económico "verde», la economía circular requiere innovación que permite ubicar la sostenibilidad ambiental en el futuro por crear y no en el pasado por mantener. Las tensiones se convierten en sinergias, que finalmente permiten seguir con el business as usual.

La economía circular podría verse como una oportunidad de seguir hablando y legislando en favor del medio ambiente y de la sostenibilidad en un momento en el que la crisis económica, el desempleo y la deuda eran las preocupaciones primarias. Por mucho que la idea de economía circular tenga sus fallos, uno puede simpatizar con las intenciones que llevaron a poner esa idea en las agendas políticas europeas. De hecho, es difícil no estar de acuerdo y articular críticas hacia una estrategia donde solo hay beneficios. Este fenómeno se ha denominado «el poder de la bondad" (Loga, 2004). Cuando se habla desde la «bondad» el discurso parece elevarse por encima de los debates políticos y crear un objetivo común con el que no se puede no estar de acuerdo. Ese tipo de políticas abre la puerta a una gestión tecnocrática y apolítica, que pueden ser muy poco democráticas. 
Al mismo tiempo, es interesante ver cómo lo que surgió como una estrategia de supervivencia se ha convertido en una de las políticas estrella del Pacto Verde Europeo. De hecho, el mismo discurso que retrata la sostenibilidad como una estrategia de crecimiento económico se reproduce en el Pacto Verde Europeo y en las políticas de «bioeconomía", otro concepto ambiguo que se refiere al uso de biomasa como estrategia para llegar a una economía más sostenible. La economía circular parecería haber sido un precursor de un nuevo discurso, ahora hegemónico, en las políticas de crecimiento «verde». En 2021, la economía circular ya no se puede considerar un compromiso que permite a los objetivos ambientales ser parte de las políticas públicas europeas, sino que ahora las contradicciones que conlleva la idea pueden hacerse importantes a la hora de implementar esas políticas en la práctica. ¿Qué pasa si las estrategias de economía circular no logran promover la sostenibilidad? ¿Qué pasa si la reutilización y el reciclaje de productos y materiales aumentan la demanda energética de la economía? ¿Qué pasa si apostar por la conversión de los residuos en recursos económicos genera un efecto rebote? Por ejemplo, el reciclaje de aceite de freír usado como combustible ha llevado a la importación de aceite de palma, cuyo uso por la industria alimentaria ha sido denunciado por la deforestación de selva asociada al cultivo de palma aceitera (Campbell, 2021). En un sistema complejo, hay tanto mecanismos de trade-off como sinergias. Hablar solo de las sinergias es un tipo de simplificación que puede crear más problemas que beneficios.

\section{Conclusiones}

La pregunta que la economía circular trata de contestar es: ¿puede la economía capitalista ser sostenible? Ese es un debate muy antiguo, que se hizo mainstream en la Cumbre de la Tierra de Río de Janeiro de 1992, en la que se popularizó el concepto de desarrollo sostenible. La economía circular es una de las últimas iteraciones de ese debate, y su respuesta es que sí, se puede resolver la tensión entre economía y ambiente. Si los recursos se hacen cada vez más escasos, la idea es reciclar, reutilizar y reparar, para que los materiales se queden en la economía el máximo de tiempo posible. Más allá del alcance limitado de la economía circular en la práctica, ya que solo se pueden recircular los recursos naturales que no sufren un proceso entrópico y no se degradan en demasía, y que la circularidad llevaría más bien a una desaceleración de la economía, el imaginario de una economía circular -o, más en general, de un crecimiento económico basado en estrategias sostenibles y verdes- crea un discurso apolítico, donde solo hay ganadores y sinergias, donde se invisibilizan los perdedores y los costes. En esta nueva variante del capitalismo, lo que está en peligro no es solo la base biofísica que sustenta la economía, sino también el debate democrático que se genera cuando hay que elegir entre diferentes estrategias y cuando hay que resolver conflictos de intereses. En la sostenibilidad, hay decisiones difíciles de tomar sobre quién debería beneficiarse y quién no, sobre los costes que puede tener una economía más sostenible, sobre los cambios que deberían sufrir los sistemas de producción y los patrones de consumo, etc. No hay que confundir el solucionar la parte incómoda de los problemas de sostenibilidad con el haber solucionado el problema en sí. 


\section{Referencias bibliográficas}

CAMPBELL, M. (2021). «Europe's reliance on used cooking oil is fueling deforestation, says new study». En: Euronews [en línea]. Disponible en: https://www.euronews.com/green/2021/04/21/europe-s-reliance-on-used-cooking-oil-isfuelling-deforestation-says-new-study [fecha de consulta: 21 de abril de 2021].

COMISIÓN EUROPEA (2015). Communication from the Commision to the European Parliament, the Council, the European Economic and Social Committee and the Committee of the Regions. Closing the loop - An action plan for the Circular Economy. COM/2015/0614. Bruselas: Comisión Europea.

GIAMPIETRO, M. (2019). «On the circular bioeconomy and decoupling: implications for sustainable growth». En: Ecological economics, núm. 162, págs. 143-156 [en línea]. DOl: https://doi.org/10.1016/j.ecolecon.2019.05.001

GIAMPIETRO, M.; FUNTOWICZ, S. O. (2020). «From elite folk science to the policy legend of the circular economy». En: Environmental Science \& Policy, núm. 109, págs. 64-72 [en línea]. DOI: https://doi.org/10.1016/j.envsci.2020.04.012

GIAMPIETRO, M.; MAYUMI, K.; SORMAN, A. (2011). The metabolic pattern of societies: where economists fall short [en línea]. Londres: Routledge. DOl: https://doi.org/10.4324/9780203635926

HAAS, W.; KRAUSMANN, F.; WIEDENHOFER, D.; HEINZ, M. (2015). «How circular is the global economy?: An assessment of material flows, waste production, and recycling in the European Union and the world in 2005». En: Journal of industrial ecology, vol. 19, núm. 5, págs. 765-777 [en línea]. DOl: https://doi.org/10.1111/jiec.12244

HALL, C. A.; KLITGAARD, K. A. (2011). Energy and the wealth of nations: understanding the biophysical economy. Springer Science \& Business Media.

KOVACIC, Z.; STRAND, R.; VÖLKER, T. (2019). The circular economy in Europe: Critical perspectives on policies and imaginaries [en línea]. Londres: Routledge. DOl: https://doi.org/10.4324/9780429061028

LOGA, J. M. (2004). Godhetsmakt. Tesis doctoral. Bergen: University of Bergen.

MARTINEZ-ALIER, J. (2016). «La economía no es circular sino entrópica». En: EconomíaSur [en línea]. Disponible en: https://economiasur.com/2016/08/la-economia-no-es-circular-sino-entropica/

MAYUMI, K.; GIAMPIETRO, M. (2019). «Reconsidering "circular economy" in terms of irreversible evolution of economic activity». En: Romanian Journal of Economic Forecasting, vol. 22, núm. 2, págs. 196-206.

VÖLKER, T.; KOVACIC, Z.; STRAND, R. (2020). «Indicator development as a site of collective imagination? The case of European Commission policies on the circular economy». En: Culture and Organization, vol. 26, núm. 2, págs. 103-120 [en línea]. DOI: https://doi.org/10.1080/14759551.2019.1699092

Cita recomendada: KOVACIC, Zora. Contradicciones y promesas de la economía circular. Oikonomics [en línea]. Noviembre 2021, n. 16. ISSN: 2339-9546.

DOI: https://doi.org/10.7238/o.n16.2114 


\section{Zora Kovacic}

zkovacic@uoc.edu

Universitat Oberta de Catalunya

Doctorada en el Instituto de Ciencias y Tecnologías Ambientales (ICTA) de la Universidad

Autónoma de Barcelona. Graduada en Economía y Desarrollo por la School of Oriental and African Studies (SOAS), máster en Estudios Ambientales por la Universidad Autónoma de Barcelona y la Universidad Tecnológica de Hamburgo. Investigadora posdoctoral en el grupo Urban Transformation and Global Change (TURBA) del Internet Interdisciplinary Institute (IN3) de la UOC. Su investigación se centra en el rol de la innovación como imaginario en las políticas públicas de sostenibilidad. Ha trabajado en Stellenbosch University (Sudáfrica) y en la Universidad de Bergen (Noruega).

Los textos publicados en esta revista están sujetos -si no se indica lo contrario- a una licencia de Reconocimiento 4.0 Internacional de Creative Commons. Puede copiarlos, distribuirlos, comunicarlos públicamente, hacer obras derivadas siempre que reconozca los créditos de las obras (autoría, nombre de la revista, institución editora) de la manera especificada por los autores o por la revista. La licencia completa se puede consultar en https://creativecommons.org/licenses/by/4.0/deed.es_ES.

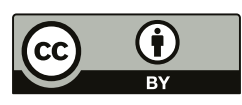

